\title{
SENDEROS SUBMARINOS DEL PARQUE NACIONAL CAHUITA: DESCRIPCIÓN BIOLÓGICA
}

\author{
Gerardo E. Palacios Martínez ${ }^{1 *}$
}

\section{RESUMEN}

Los recorridos en los Senderos Submarinos del Parque Nacional Cahuita permitieron identificar los organismos marinos presentes. La descripción biológica de la flora y la fauna se basó en la metodología de línea transecto y observación directa. El Sendero Submarino Perezoso presentó un $40 \%$ de cobertura de coral vivo, entre los cuales estaba el coral estrella (Siderastrea siderea) como dominante, las especies de peces más frecuente pertenecen a la familia Pomacentridae. El erizo negro (Diadema antillarum) es el más frecuente de los invertebrados y las algas registradas son del género Dictyota y la especie Halimeda opuntia. Las especies de coral Siderastrea siderea y Agaricia agaricites son las más frecuentes en los senderos submarinos de Perezoso y Eduardo.

Palabras claves: Descripción biológica, sendero submarino, Cahuita.

\begin{abstract}
This survey provides biological information regarding the underwater trails of Cahuita National Park, and identifies the most common species present. The biological description of the flora and fauna are based on line transects and direct observation. The Perezoso underwater trail had $40 \%$ live coral cover, of which the Lobe coral (Siderastrea siderea) was the most common; the most common species of fish belonged to the family Pomacentridae. The long spined sea urchin (Diadema antillarum) was the most common among the invertebrates, and the algae recorded belonged to the genera Dictyota and Halimeda. The coral species Siderastrea siderea and Agaricia agaricites were the most common corals of the Perezoso and Eduardo underwater trails.
\end{abstract}

Keywords: Biological description, underwater trail, Cahuita.

1 Fundación Promar (PROMAR) - Centro Científico Tropical (CCT), San José, Costa Rica, gpalacios@ fundacionpromar.org*, gerarp2003@hotmail.com*

Recibido 18-V-2009

Aceptado 08-X-2009

DOI: http://dx.doi.org/10.15359/revmar.1.13

Rev. Mar. y Cost. ISSN 1659-455X. Vol. 1. 231-244, Diciembre 2009. 


\section{INTRODUCCIÓN}

El Parque Nacional Cahuita se creó con el objetivo de preservar y proteger el arrecife coralino más importante de Costa Rica, el bosque tropical húmedo basal y la belleza escénica de sus playas (Piedrahita, 1997).

El Parque incluye el arrecife de coral marginal más largo y diverso sobre la costa Atlántica de Costa Rica con 34 especies de corales hermatípicos escleractinios (Guzmán y Cortés, 1985a), 19 especies de octocoralios (Guzmán \& Cortés, 1985b), 6 especies de corales zooxantelados (Cortés \& Jiménez, 2003, citados en Wychoff \& Collage, 2004) y 3 especies de hidrocorales (Cortés, 1992, citado en Wychoff \& Collare, 2004).

Los arrecifes del Parque se han estudiado sistemáticamente desde los años setenta. Este ecosistema presenta altos niveles de degradación. Durante 23 años, entre 1970 y 1993, la cobertura de coral vivo pasó de $40 \%$ a 11\% (Ramírez, 1993, citado en Piedrahita, 1997). El Parque Nacional Cahuita cuenta con un inventario marino de 128 especies de algas, 44 de crustáceos, 140 de moluscos y 123 de peces.

Este estudio pretendió realizar una descripción general actualizada de la diversidad biológica de los senderos submarinos y otros ecosistemas de uso turístico presentes en el Parque.

\section{MATERIALES Y MÉTODOS}

\section{Descripción de los Senderos Sub- marinos de Perezoso y Eduardo}

Los días 3, 4 y 11 de abril del 2006 se realizaron tres recorridos a lo largo de cada uno de los senderos submarinos con equipo básico (snorkeling) anotando las especies presentes de flora y fauna. El área del recorrido estaba determinada por la longitud de cada sendero, el Perezoso midió 192 $\mathrm{m}$, mientras que el otro sendero midió $508 \mathrm{~m}$ con un ancho de observación de tres metros. Las anotaciones se realizaron por observaciones directas y registradas en una tabla acrílica in situ. Además, se realizó un recorrido al lado de la línea de boyas que demarcan los senderos submarinos de Perezoso y Eduardo con un GPS Magellan ${ }^{\circledR} 315$. Los datos se procesaron en Arc GIS para obtener el mapa de estos basado en fotos aéreas georreferenciadas; con esta información se logró determinar la longitud de los senderos.

Se identificaron los organismos presentes en los senderos submarinos por medio de las guías de campo de Greenberg (1986) y de Kaplan (1988). A las 9:00 a.m. del 11 de abril del 2006, por medio del uso del equipo SCUBA, se dispuso una línea transecto de 10 metros a lo largo del bentos del Sendero Submarino Perezoso, para medir los distintos sustratos presentes. Se identificó el tipo de sustrato, la cantidad de centímetros que ocupaba cada sustrato y cada medición se anotó en una tabla acrí- 
lica in situ todo el proceso se realizó durante 60 minutos.

\section{Decápodos de Playa Blanca}

Se evaluó la densidad de huecos con un diámetro superior a medio centímetro, confeccionados por los cangrejos marinos, principalmente Ocypode spp, en la zona entre mareas en los fondos suaves. Se realizaron seis líneas transectos perpendiculares a la playa, consecutivas y separadas una de la otra por cien metros.

Tres transectos estaban ubicados frente a las banderas verdes y los otros frente a las banderas rojas. Cada transecto se dispuso en la parte entre mareas donde se encontraban las cavernas hechas por los decápodos. Luego se dispuso un cuadrante de un metro cuadrado de forma alterna y consecutiva a lo largo de cada línea transecto de diez metros.

\section{Área de Estudio}

El Parque Nacional Cahuita ( $9^{\circ}$ $45^{\prime}$ Norte $-82^{\circ} 48^{\prime}$ Este) está ubicado en el Área de Conservación La Amistad Caribe (ACLAC), cantón de Talamanca, provincia de Limón, a 45 $\mathrm{km}$ al sureste de la ciudad de Limón (Piedrahita, 1997).

\section{Sendero Submarino Perezoso}

Este sendero se ubica en el punto Noreste con coordenadas geográficas $9^{\circ} 45^{\prime} 2.63$ ” N y $82^{\circ} 49^{\prime} 16.75^{\prime \prime}$ E, si- gue una línea recta hasta el punto Suroeste de coordenadas geográficas $9^{\circ}$ $45^{\prime} 1.42^{\prime \prime} \mathrm{N}$ y $82^{\circ} 49^{\prime} 22.59^{\prime \prime}$ E (Figura 1). Se encuentra dentro del Parque Nacional Cahuita, frente a la desembocadura del Río Perezoso, de forma paralela a la playa en una zona típica de arrastre de troncos provenientes de la parte continental. Actualmente, el sendero está demarcado por una línea de boyas en su lado costero, abarcando parte de la cresta (0.5-1.5 m de profundidad). La extensión longitudinal del sendero es de 192 metros.

El sendero comprende solo la parte del arrecife coralino especialmente sobre la cresta arrecifal. Entre la playa y el sendero se encuentra el ecosistema de pastos marinos. Este sendero es el que sufre de forma más directa la acción de los ríos Estrella y Banano por su ubicación geográfica. La única forma para llegar al sendero es en bote, no se puede llegar por la playa.

\section{Sendero Submarino Eduardo}

Este sendero con forma de herradura pasa por el punto Oeste, cuyas coordenadas geográficas son $9^{\circ} 44^{\prime}$ $23.96 " \mathrm{~N}$ y $82^{\circ} 48^{\prime} 31.30^{\prime \prime} \mathrm{E}$, sigue hasta el punto Norte de coordenadas geográficas $9^{\circ} 44 " 26.94 " \mathrm{~N}$ y $82^{\circ}$ 48 '27.7" E, sigue rodeando hasta llegar al punto Este, cuyas coordenadas son $9^{\circ} 44^{\prime} 24.56^{\prime \prime} \mathrm{N}$ y $82^{\circ} 48^{\prime}$ 25.72 " E y el punto Sur sus coordenadas son $9^{\circ} 44^{\prime} 21.70^{\prime \prime} \mathrm{N}$ y $82^{\circ}$ $48^{\prime} 28.55$ " E (Figura 1). En la fecha 


\section{Figura 1}

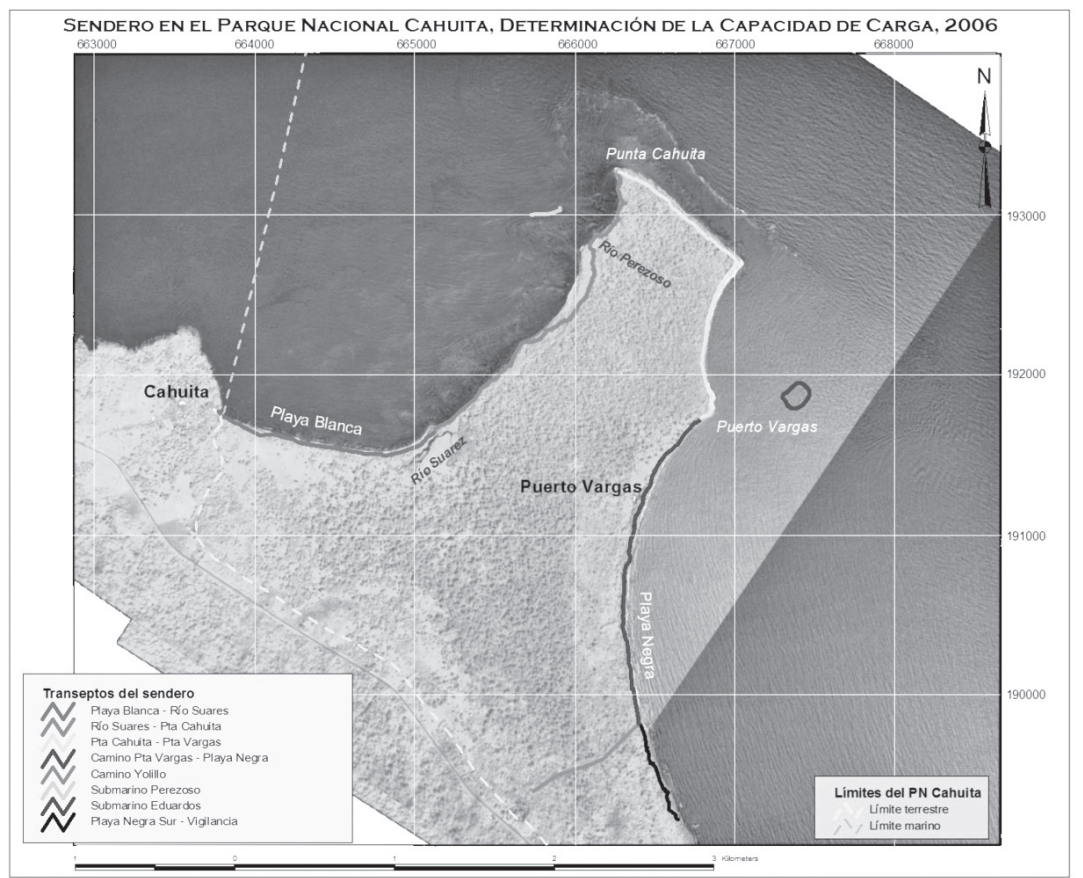

Ubicación geográfica de los senderos submarinos Perezoso, Eduardo y de Playa Blanca.

Location of underwater trails, Perezoso, Eduardo, and Blanca Beach.

de este estudio, el sendero estaba fiere su forma redondeada, permidemarcado por dos líneas de boyas tiendo a los visitantes salir y llegar en forma de herradura, cercando un arrecife coralino que se encuentra entre 1-4 $\mathrm{m}$ de profundidad. La extensión longitudinal del sendero es de 508 metros y el ancho aproximado es de $8 \mathrm{~m}$.

El sendero se encuentra en dirección Noreste desde Punta Cahuita frente a la playa de Puerto Vargas. Las corrientes que llegan a esta zona son más fuertes, comparadas con las corrientes del Sendero Submarino Perezoso. El sendero surca el arrecife coralino, lo que le concerca de la zona donde está fondeado el bote.

\section{Playa Blanca}

Esta playa se ubica en el punto Suroeste, cuyas coordenadas geográficas son $9^{\circ} 44^{\prime} 20.70^{\prime \prime} \mathrm{N}$ y $82^{\circ} 50^{\prime}$ 26.47" E y el punto Noreste, cuyas coordenadas son $9^{\circ} 44^{\prime} 17.53^{\prime \prime} \mathrm{N} \mathrm{y}$ $82^{\circ} 49^{\prime} 42.64$ ” E (Figura 1).

Esta playa está constituida por fondos suaves con alta proporción de arena. Los organismos más 
abundantes y dominantes son los decápodos, principalmente cangrejos y moluscos que habitan este ecosistema. Está limitada por el ecosistema de pastos marinos por el mar, el Río Suárez al Noreste, la entrada del Parque por Cahuita al Suroeste y el sendero de entrada a la playa paralelo a esta con una separación de una pequeña franja de bosque de escasos metros.

Playa Blanca se ha dividido en dos partes. Una demarcada con banderas rojas que indican la imposibilidad de nadar por las fuertes corrientes y la demarcada con banderas verdes que dan vía libre a los nadadores para usar esa parte de la playa. Esta división se da en el punto cuyas coordenadas son $9^{\circ} 44^{\text {' }}$ 15.44 ” N y $82^{\circ} 50$ ' 8.09 ” E.

\section{RESULTADOS}

\section{Descripción de los senderos}

Sendero Submarino Perezoso. El sendero submarino tiene como objetivo la observación del arrecife de tipo costero, principalmente en la diversidad que presenta este ecosistema cerca de su cresta. El transecto realizado en el Sendero Submarino Perezoso presentó un $40 \%$ de cobertura de coral vivo en los $10 \mathrm{~m}$ iniciales. Las especies de coral vivo que sustentan el arrecife están dominadas principalmente por el coral estrella (Siderastrea siderea), seguida por el coral lechuga (Agaricia agaricites) y la abundancia más baja está representada por el coral cerebro (Diploria strigosa) y el hidroide calcáreo (Millepora complanata, Figura 2). Otras especies

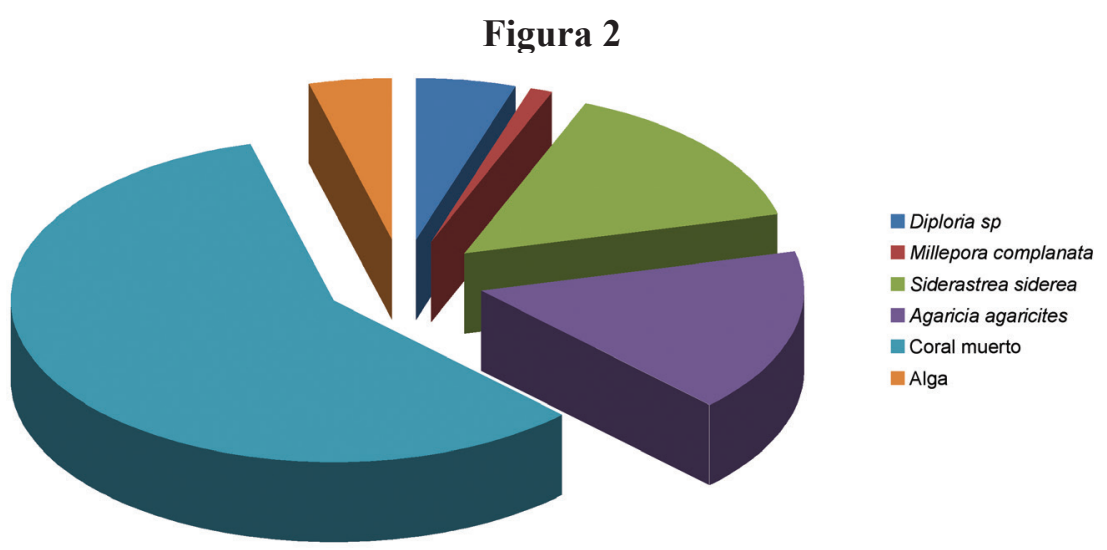

Porcentajes de la composición bentónica del Sendero Submarino Perezoso dentro de la línea transecto de $10 \mathrm{~m}$ de largo.

Benthic percentage composition at the Perezoso underwater trail within the $10 \mathrm{~m}$ transect. 
de coral encontradas en el sendero son Acropora palmata y con relativa frecuencia Stephanocoenia intersepts.

Fauna Íctica. La fauna íctica predominante en el sendero se centra en especies neríticas. Las especies más abundantes son de la familia Pomacentridae (damiselas), principalmente, Pomacentrus fuscus, Pomacentrus variabilis, Abudefduf saxatilis y Microspathodon chrysurus (Figura 3). El total de especies observadas fue de 32 .

También se observaron especies de las familias Holocentridae, Labridae, Acanthuridae, Carangidae, Opistognathidae, Scaridae, Chaetodontidae, Synodontidae y Haemulidae. Las especies registradas de estas familias son: Bodianus rufus, Acanthurus bahianus, Acanthurus coeruleus, Caranx sp, Ophioblen- nius atlanticus, Elagatis bipinnulata, Thalassoma bifasciatum, Halichoeres maculipinna, Sparisoma viride, Chaetodon striatus, Chaetodon ocellatus, Haemulon aurolineatum y Anisotremus virginicus.

Otras especies observadas son: Chaetodipterus faber (Ephippidae), Pomacanthus paru (Pomacanthidae), Lactophrys sp (Ostraciidae), Haemulon sp, Haemulon sp2, Gymnothorax funebris (Muraenidae), Lutjanus mahogoni y Lutjanus sp (Lutjanidae), Chaetodon sp, Narcine brasiliensis (Narcinidae), Echeneis sp (Echeneidae), Lutjanus sp, Holocentrus sp, Diodon hystrix (Diodontidae) y Epinephelus cruentatus (Serranidae).

Fauna de Invertebrados Marinos. Los macroinvertebrados bentónicos observados fueron el erizo de mar,

\section{Figura 3}

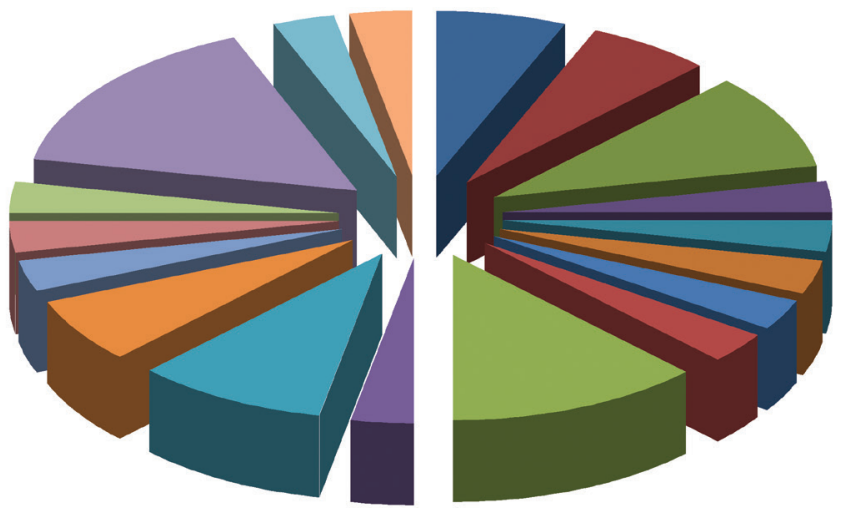

$$
\begin{aligned}
& \text { - Acanthuridae } \\
& \text { - Carangidae } \\
& \text { Chaetodontidae } \\
& \text { - Dasyatidae } \\
& \text { - Diodontidae } \\
& \text { Echeneidae } \\
& \text { Ephippidae } \\
& \text { - Ginglymostomatidae } \\
& \text { Haemulidae } \\
& \text { - Labridae } \\
& \text { - Lutjanidae } \\
& \text { Muraenidae } \\
& \text { - Opistognathidae } \\
& \text { - Ostraciidae } \\
& \text { In Pomacanthidae } \\
& \text { ncaridae } \\
& \text { - Serranidae }
\end{aligned}
$$

Riqueza íctica de las familias observadas en los senderos submarinos. Richness in fish families recorded in the underwater trails. 
principalmente el erizo negro (Diadema antillarum), también se encontraron otras dos especies como Tripneustes ventricosus y Eucidaris tribuloides.

El resto de invertebrados con registros muy esporádicos fueron algunos crustáceos como la langosta espinosa (Panulirus argus), el cangrejo flecha (Stenorhynchus seticornis) y el camarón payaso (Stenopus hispi$d o s)$. Además se encontró el gusano de fuego (Hermodice carunculata), hidroides, esponjas, octocorales, anémonas, gastrópodos, la estrella quebradiza (Ophioderma sp), la estrella de mar (Linckia sp) y el arbolito de navidad (Spirobranchus giganteus) (Figura 4).

Flora Algal. A lo largo del sendero se observaron parches de algas principalmente de los géneros Dictyota y Halimeda.

Sendero Submarino Eduardo. Este sendero submarino, al igual que su similar en Perezoso, tiene como objetivo la observación del arrecife de tipo costero. Los corales y otros organismos asociados, analizados en este estudio, mantienen una composición bastante parecida entre los dos senderos. En las observaciones directas en Eduardo se observó una fauna íctica de mayor tamaño y en grupos más numerosos.

Las especies de coral que sustentan el arrecife en este sendero son muy similares a las de Perezoso, principalmente por el coral estrella (S. siderea), el coral lechuga (A. agaricites) y el coral cerebro (D. strigosa), también se registró el hidroide calcáreo

Figura 4

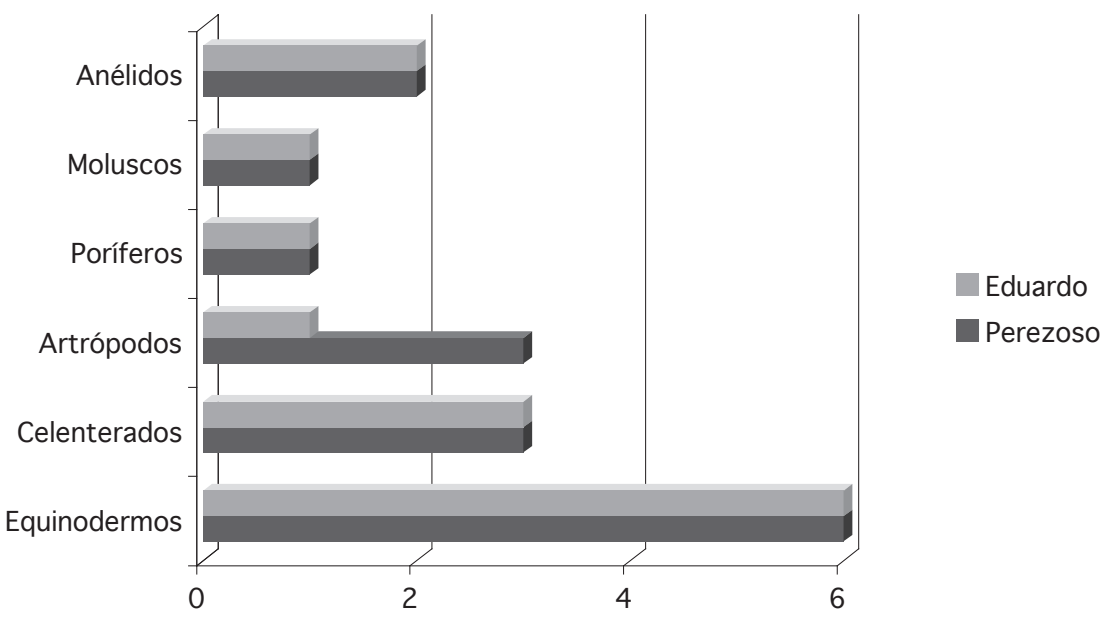

Comparación entre el número de especies de invertebrados y cada sendero.

Comparison of number of invertebrate species among underwater trails. 
(M. complanata). Otras especies de coral encontradas en el sendero son A. palmata y Stephanocoenia intersepts, así como el octocoral Pseudopterogorgia sp.

Fauna Íctica. La fauna íctica en el sendero Eduardo coincidió con la mayoría de las especies encontradas en el sendero Perezoso, con un total de 33 especies. El tiburón nodriza (Ginglymostoma cirratum) hizo la diferencia.

Fauna de Invertebrados Marinos. Los macroinvertebrados bentónicos están representados de manera similar al sendero Perezoso; el cangrejo flecha (Stenorhynchus seticornis) y el camarón payaso (Stenopus hispidos), únicamente se encontraron en Perezoso y no en Eduardo (Figura 4).

Flora Algal. A lo largo del sendero se observan parches de algas principalmente de los géneros Dictyota, Avrainvillea, Galaxaura y Halimeda.

Playa Blanca. El área total de este sector de playa desde el inicio del Parque hasta el Río Suárez es de $7.291 \mathrm{~m}^{2}$, de los cuales aproximadamente la mitad está demarcada por banderas rojas y verdes. Las banderas de color rojo señalan el impedimento a los bañistas para nadar en ese lugar, debido a las corrientes. Esta condición indirectamente protege la fauna y flora marina del lugar.

Las banderas de color verde autorizan a los bañistas para nadar en ese lugar, ya que posee aguas cal- madas. Las características biológicas de la playa están definidas por dos ecosistemas, uno de ellos son los fondos blandos con mayor proporción de arena y las fanerógamas marinas.

En los fondos blandos se observaron decápodos, principalmente cangrejos del género Ocypode. Otro grupo de invertebrados presentes son los moluscos, sobre todo de gastrópodos, estos últimos son fáciles de observar sobre troncos.

Fauna de Invertebrados Marinos. La densidad de huecos de cavernas de cangrejos indicó una mayor densidad, con un promedio de $25 \pm$ 6 agujeros en la zona de las banderas rojas. En la zona de playa con banderas verdes se encontró una densidad promedio de $14 \pm 2$ agujeros.

Otro invertebrado bentónico observado en esta área fue una especie de pepino común sin identificar, pero además se observó la presencia de la langosta espinosa ( $P$. argus), el gastrópodo Strombus gigas y gran cantidad de hidroides epibiontes en las hojas del pasto tortuga.

Flora Marina. Frente a Playa Blanca, en el área marina cerca de la costa, se observaron praderas de fanerógamas, principalmente compuestas por Thalassia testudinum (pasto tortuga), Syringodium filiforme (pasto manatí).

Las especies algales observadas en la visita a los pastos marinos de Playa Blanca están representadas por los géneros Padina, Sargassum, Caulerpa, 
Laurencia, Bryopsis, Amansia, Dictyota, Avrainvillea y Galaxaura. Además se encontraron las especies: Amphiroa fragilissima, Caulerpa racemosa, Caulerpa sertularioides, Dictyota bartayresiana, Enteromorpha flexuosa y Halimeda opuntia.

\section{DISCUSIÓN}

\section{Arrecifes Coralinos de los Sende- ros Submarinos}

Las especies Siderastrea siderea y Agaricia agaricites son las dominantes en los senderos submarinos de Perezoso y Eduardo, lo que coincide con los estudios realizados por Cortés (1992) y Wychoff \& Collage (2004). La conformación típica del arrecife coralino de Cahuita corresponde a la de arrecifes marginales, los cuales son barreras calcáreas construidas por organismos, paralelas y cercanas a la costa (Cortés y Risk, 1984, citados en Cortés, 1992).

El valor de cobertura de $A$. agaricites en este estudio fue similar al encontrado por Wychoff \& Collage (2004). Mientras que el valor de $S$. siderea es más bajo en este estudio con respecto al registrado por Wychoff \& Collage (2004).

Este último valor se puede explicar a que es un valor promedio en una zona muy puntual como es el sendero, puede que en otras partes del arrecife los valores sean mayores. Otra razón es la presión que puede estar ejerciendo el turismo sobre la cobertura de coral. La disminución en el porcentaje de este coral se ha observado en otros estudios desde 1993 hasta el 2004, debido al aporte de contaminantes y sedimentos por los ríos cercanos (Cortés, 1994, citado en Wychoff \& Collage, 2004).

La baja abundancia observada en el sendero Perezoso de A. palmata, Montastrea annularis y Acropora cervicornis, hace difícil la recuperación del arrecife, ya que son agentes reconstructores de este ecosistema. Esta condición puede ser producto de las malas prácticas de manejo establecidas en las áreas marino-costeras (Veron, 1992).

Desde la introducción de los monocultivos de banano y la deforestación a la que se sometió el Caribe Sur de Costa Rica, los arrecifes coralinos de Cahuita y zonas aledañas han demostrado un fuerte impacto con una alta mortalidad de corales y degradación del ecosistema en general. En las últimas dos décadas se ha incrementado la sedimentación en las aguas que circundan el Parque Nacional Cahuita afectando sensiblemente la salud de todos sus ecosistemas marinos (Wychoff \& Collage, 2004).

Cortés (1989), citado en Cortés (1992), afirma que los arrecifes costeros someros en Limón están siendo afectados por sedimentos terrígenos, producto de la deforestación de la zona costera y de las cuencas de riachuelos del área (Rogers, 1990, citado en Wychoff \& Collage, 2004). 
Estos cambios en el arrecife se dan en cortos y largos lapsos, empezando con la fauna herbívora como los erizos de mar. Lo más preocupante es el cambio de las colonias coralinas por parches de algas (Birkeland, 1997). Estos cambios de dominancia de corales reemplazados por algas comúnmente no son reversibles. Se ha comprobado con décadas de seguimientos realizados en diferentes partes del Caribe (Hughes, 1994).

Los parches de Halimeda opuntia observados en el sendero Perezoso dificultan el desarrollo del coral (Rogers, 1979, citado en Wychoff \& Collage, 2004). Esta alga se ve beneficiada por la baja gradual en la densidad de especies herbívoras de Cahuita, como el erizo de mar (D. antillarum) (Birkeland, 1997). Esta condición causa la baja de la abundancia de las colonias coralinas del parque.

La presencia del poliqueto Hermodice carunculata puede explicar la reducción del hidroide Millepora complanata. Sin embargo, la presencia de este anélido en el sendero es muy baja, por eso su influencia llega a ser despreciable (Ott \& Lewis, 1972; Nowlton, 1992).

Con respecto al porcentaje de coral vivo presente en este estudio del sendero Perezoso está dentro de los rangos reportados por Wychoff \& Collage (2004). El porcentaje de coral vivo cambia dependiendo de las diferentes zonas dentro del mismo Parque, encontrando el valor de $40 \%$ como uno de los más altos. Teniendo en cuenta estudios realizados por Cortés (1994), citado en Wychoff \& Collage (2004), este 40\% era frecuente en los años setenta, pero actualmente los promedios se acercan a valores del 11\%. Según Wychoff \& Collage (2004), $25.8 \%$ es el porcentaje promedio del arrecife cahuiteño.

Diversos estudios en comunidades coralinas revelan que el grado de recuperación de los corales es mucho menor después de alteraciones debidas a agentes externos, que en condiciones normales (Birkeland, 1997). Esta aseveración debe tomarse en cuenta para evitar seguir afectando el arrecife cahuiteño con las distintas prácticas que están produciendo su mortalidad.

\section{Fauna Íctica}

La fauna íctica es susceptible al estado de salud de los arrecifes coralinos, presentando bajas densidades y diversidades (Perez-Cruet, 1984, citado en Wychoff \& Collage, 2004), por esto la discusión sobre los problemas que enfrenta en la actualidad el arrecife coralino de Cahuita afecta directamente a la población de peces; muchos de ellos presentan valores de abundancia más bajos de lo normal.

La semejanza en el número de especies de peces entre este estudio y el realizado por Wychoff \& Collage (2004) puede deberse a que las condiciones en el arrecife no han cambiado, tanto en el ecosistema como en la presencia de sedimentos y contaminantes. 
En el Parque Nacional Cahuita es frecuente observar al tiburón nodriza (Ginglymostoma cirratum), el cual está incluido en la lista roja de especies con algún grado de amenaza publicada por la UICN (2006). Esta especie no tiene datos suficientes para hacer una evaluación de su riesgo de extinción de forma directa o indirecta, basándose en su abundancia, distribución y/o su estado poblacional.

Este tiburón está clasificado como DD (Datos Deficientes), por lo tanto, no es una categoría de amenaza, pero indica que es muy factible que con los datos de investigaciones futuras se demuestre que este pez cartilaginoso es una especie amenazada. Los estudios preliminares de su biología indican su fuerte arraigo al sitio donde habita, lo que hace al nodriza muy vulnerable a la extracción local por sobrepesca (Compagno, 2001, citado en UICN, 2006).

\section{Fauna Invertebrada}

Existen muy pocos estudios ecológicos sobre invertebrados bentónicos de la zona, aunque muchos de estos organismos son importantes dentro del equilibrio del ecosistema en general. Entre los pocos estudios realizados en Cahuita se encuentra el realizado por Wehrtmann (2004) sobre la langosta espinosa (Panulirus argus), el cual se basó en encuestas realizadas a pescadores.

Las capturas de este crustáceo en Cahuita se han reducido en los últi- mos diez años. Los pescadores con quince años de experiencia en la zona lo atribuyen a la pesca mal regulada y a la contaminación. Este resultado puede explicar la baja frecuencia de observación en los senderos submarinos.

En las observaciones hechas en campo en este trabajo, se observó a pescadores de langosta extrayendo individuos de escaso tamaño (Figura 5). Esta observación coincide con los resultados obtenidos por Wehrtmann (2004) en su estudio. Los pescadores de langosta de Cahuita, Manzanillo y algunos de Puerto Viejo pescan un $50 \%$ de las langostas por debajo de su talla legal. Esto causa problemas, ya que eliminan parte de los futuros reproductores de la población.

Otros decápodos importantes en la zona son los encontrados en Playa Blanca, especialmente del género Ocypode. Los resultados efectuados en cuanto a la densidad de cangrejos en la playa indicaron mayor abundancia de estos decápodos en la zona de las banderas rojas comparada con las verdes. Esta situación es debida probablemente a que el mayor uso de la gente en esta playa disminuye la base alimenticia de estos cangrejos y perturban continuamente su comportamiento al tener que esconderse por la presencia humana.

Al incidir en el comportamiento de los cangrejos se afecta todo el ecosistema. El ave migratoria Calidris alba, conocida vulgarmente como patudo, se alimenta de este crustáceo cuando baja la marea, pero cuando 


\section{Figura 5}

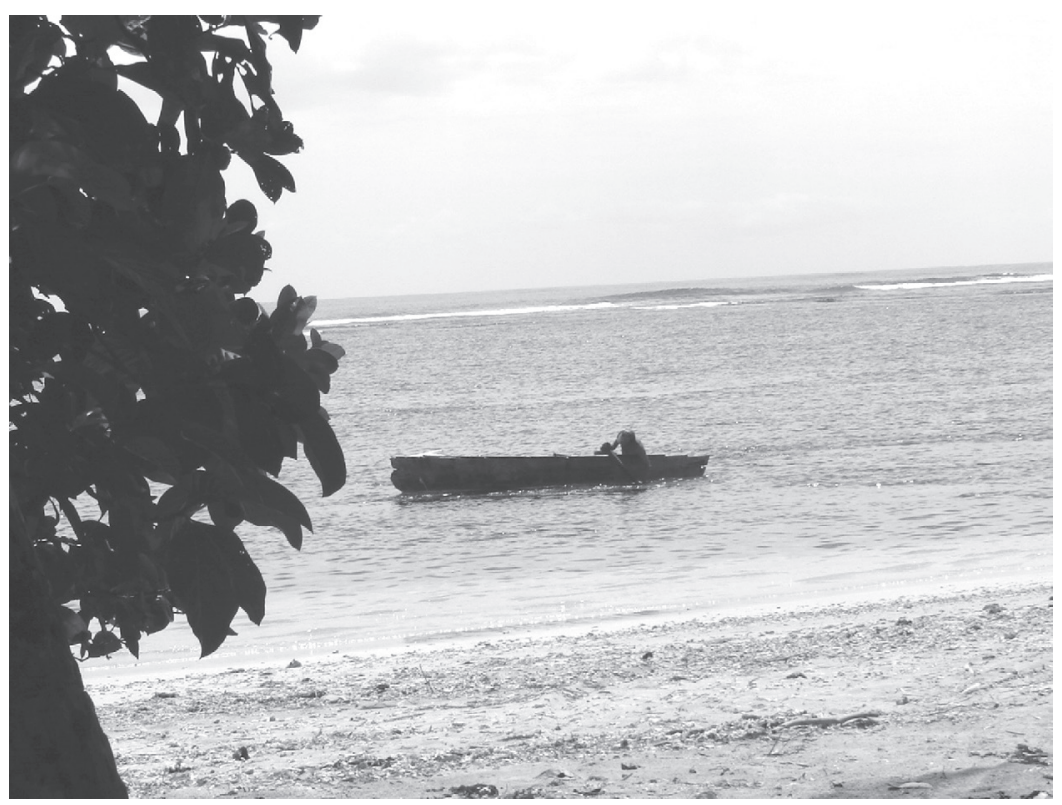

Pescador artesanal de langostas dentro del Parque Nacional Cahuita. Fotografía: V. Jiménez. Artisanal lobster fisherman, fishing inside Cahuita National Park. Picture: V. Jiménez.

la marea sube este crustáceo sirve de alimento para una amplia variedad de peces (Little, 2000).

\section{Flora Algal}

Las algas observadas en este estudio en los senderos submarinos son las mismas que las encontradas por Wychoff \& Collage (2004), las cuales son Dictyota sp y Halimeda opuntia. También coincide con las observaciones hechas por Garzón (s.f.), el cual encuentra que el alga $H$. opuntia es muy abundante y se encuentra colonizando diferentes sustratos vivos.

\section{CONCLUSIONES}

- Las especies de coral Siderastrea siderea y Agaricia agaricites son las más frecuentes en los senderos submarinos de Perezoso y Eduardo.

- La división de Playa Blanca en la zona de las banderas rojas ha beneficiado indirectamente a la población de cangrejos y otro tipo de fauna bentónica.

\section{AGRADECIMIENTOS}

Agradezco el apoyo brindado a esta evaluación por The Nature Conservancy (TNC), al financiar el proyecto al grupo consultor de Fundación Promar y Centro Científico 
Tropical y al personal del Parque Nacional Cahuita.

\section{BIBLIOGRAFÍA}

Birkeland, C. (1997). Introduction. 1-12 pp. En: Birkeland, C. Life and death of coral reefs. Chapman \& Hall, ITP (Ed.). USA.

Cortés, J. (1992). Los Arrecifes Coralinos del Refugio Nacional de Vida Silvestre Gandoca-Manzanillo, Limón, Costa Rica. Rev. Biol. Trop., 40(3), 325-333.

Garzón, J. (s.f.). Bahía de Chengue, Parque Natural Tayrona, Colombia. UNESCO. Extraído en julio de 2006. http://www.unesco. org/csi/pub/papers/garzon.htm

Greenberg, I. (1986). Guide to corals $\&$ fishes of Florida, the Bahamas and the Caribbean. Haff-Daugherty Graphics, Inc (Ed.). USA.

Guzmán, H. y Cortés, J. (1985a). Organismos de los arrecifes coralinos de Costa Rica. III. Descripción y distribución geográfica de corales escleractinios (Cnidaria: Anthozoa: Scleractinia) de la costa Caribe. Brenesia, 24, 63-123.

Guzmán, H. y Cortés, J. (1985b). Organismos de los arrecifes coralinos de Costa Rica. IV. Descripción y distribución geográfica de octocoralios (Cnidaria: Anthozoa) de la costa Caribe. Brenesia, $24,125-173$.

Hughes, T. (1994). Catastrophes, phase shifts, and large-scale de- gradation of a Caribbean coral reef. Science, 265(5178), 15471551.

Jiménez, C. (2006). Com. per. Investigador del CIMAR-UCR.

Kaplan, E. (1988). Southeastern and Caribbean Seashores. Peterson Field Guides (Ed.). Library of Congress. USA.

Little, C. (2000). The biology of soft shores and estuaries. Oxford University Press. USA.

Nowlton, N. (1992). Thresholds and multiple stable states in coral reef community dynamics. Am. Zool., 32(6), 674-682.

Ott, B. \& Lewis, J. (1972). The importance of the gastropod Coralliophila abbreviate (Lamarck) and the polychaete Hermodice carunculata (Pallas) as coral reef predators. J. Can. Zool. 50(12), 1651-1656.

Piedrahita, J. (1997). El arrecife coralino de Punta Cahuita: origen de un pueblo y un parque nacional, experiencias para el desarrollo sostenible. Edit. La Nación S.A., San José.

UICN. (2006). Red List of Threatened species. Extraído en julio de 2006. http://www.iucnredlist.org/search/details.php?species $=60223 \&$ tab $=$ summ

Veron, J. (1992). Conservation of biodiversity: a critical time for the hermatypic corals of Japan. Coral Reefs, 11, 13-21.

Wehrtmann, I. (2004). Informe final, proyecto langosta: El recurso lan- 
gosta y su vinculación con la co- Wychoff, C. \& Collage, R. (2004). munidad del Caribe de Costa Rica: The effects of terrigenus sedimenun estudio multidisciplinario del tation on the health of the coral Área de Conservación Amistad ref. at Cahuita National Park, Caribe. Esc. de Biol. UCR. Costa Costa Rica. ACM tropical field Rica. research (Advisor: Jorge Cortés). CIMAR. UCR. Costa Rica. 